\title{
Incidência de fungos em pós-colheita de banana 'Prata anã'(Musa AAB)*
}

\author{
Wilson da Silva Moraes ${ }^{1}$, Laércio Zambolim² e Juliana D. Lima ${ }^{3}$
}

${ }^{1}$ Pólo Regional da APTA Vale do Ribeira, Agência Paulista de Tecnologia de Agronegócios/APTA. ²Departamento de Fitopatologia / Universidade Federal de Viçosa / UFV; ${ }^{3}$ Unidade Diferenciada de Registro / Universidade Estadual Paulista / UNESP.

* Parte da Tese de Doutorado do primeiro autor apresentada à Universidade Federal de Viçosa.

Autor para correspondência: Wilson da Silva Moraes. <wilson@aptaregional.sp.gov.br>

Data de chegada: 27/07/04. Aceito para publicação em: 07/07/05.

\begin{abstract}
Moraes, W. S.; Zambolim, L.; Lima, J. D. Incidence of mushroons in post harvest of banana (Musa spp.) 'Prata Anã' (AAB). Summa Phytopathologica, v. 32, n. 1, p. 67-70, 2006.

The objetive of this work were to determine the occurrence and frequency of fungus in 'Prata anã' banana and to elucidate the causal agent of post harvest rots on fruits from Jaíba, Minas Gerais, Brazil. Two isolation methods were used: diluition in placs from whashed green fruits and direct of mature fruits. The fungus Colletotrichum musae, Trichoderma harzianum, Fusarium equisetii, Penicillium sp., Aspergillus parasiticus, Trichothecium roseum, Colletotrichum acutatum, Alternaria sp., Cladosporium musae and Curvularia lunata were the more freqüentilly associated to fruits. The fungus

micellium discs. Colletotrichum musae showed lesioned average area arround of inoculation point equal to $5,8 \mathrm{~cm}^{2}$, while in the remaining of fungus tested the lesioned average area were smaller than 1,50 $\mathrm{cm}^{2}$. This results showed that $C$. musae is the primary agent of examined fruits rotteness, with $100 \%$ of incidence while the remaining of fungus were limited to attach the injury arround of inoculation point. The latent infection mode caused by $C$. musae favour firsty the tissue external colonization and after the opportunists fungus accelerate the fruits and crown rotteness.
\end{abstract} pathogenicity were tested by substituition of green fruits pell discs for

Additional keywords: quiescent infection, disease, colletotrichum, antracnose.

\section{RESUMO}

Moraes, W. S.; Zambolim, L.; Lima, J. D. Incidência de fungos em pós-colheira de banana (Musa spp.) 'Prata anã' (AAB). Summa Phytopathologica, v. 32, n. 1, p. 67-70, 2006.

Este trabalho teve por objetivo determinar a ocorrência e a frequiência de fungos em banana 'Prata anã' e elucidar o agente causal das podridões em pós-colheita de frutos provenientes do norte de Minas Gerais. Dois métodos de isolamento foram adotados: diluição em placas, a partir da lavagem de frutos verdes, e direto de frutos maduros. Os fungos Colletotrichum musae, Trichoderma harzianum, Fusarium equisetii, Penicillium sp. Aspergillus parasiticus, Trichothecium roseum, Colletotrichum acutatum, Alternaria sp., Cladosporium musae e Curvularia lunata foram os mais freqüentemente associados aos frutos. A patogenicidade desses fungos foi testada pela substituição de discos da casca de frutos verdes por discos de micélio. Colletotrichum musae apresentou área média lesionada em torno do ponto de inoculação igual a $5,8 \mathrm{~cm}^{2}$, enquanto para os demais fungos testados não passou de $1,50 \mathrm{~cm}^{2}$. Os resultados mostraram que $C$. musae é o agente primário das podridões dos frutos examinados com $100 \%$ de incidência e os demais fungos limitaram-se a necrosar os ferimentos em torno do ponto de inoculação. O modo de infecção latente, causada por $C$. musae, parece favorecer, primeiramente, a colonização interna dos tecidos e, posteriormente, a ação dos fungos oportunistas, que aceleram as podridões nos frutos e na coroa.

Palavras-chave adicionais: infecção quiescente, doença, colletotrichum, antracnose.

A identificação correta do agente causal das doenças cons-

titui a base para a estratégia de controle. O desenvolvimento 
dessas estratégias requer o conhecimento do patógeno, o tipo de inóculo, a forma de dispersão, o modo e momento em que a infecção ocorre e os mecanismos do hospedeiro que regulam a infecção (Johnson \& Sanghote (8)).

Nem todos os patógenos causam sérias perdas em todos os frutos. As diferenças na suscetibilidade dos frutos para a maioria dos patógenos depende das defesas do hospedeiro, da disponibilidade do inóculo e das condições ambientais. Muitos fungos que se estabelecem em frutos limitam a infecção sobre ou sob a cutícula, dentro do epicarpo ou pedicelo, ou em tecidos florais remanescentes em algum momento durante o desenvolvimento do fruto (Swinburn (19); Agrios (1); Kays (11); Johnson \& Sanghote (8)).

De modo geral, os cachos de banana, quando colhidos, são despencados no próprio local e transportados em caixas empilhadas sobre caminhões até ao mercado consumidor ou centrais de abastecimento. Neste trajeto, os frutos estão sujeitos a danos ou injúrias mecânicas que podem ocorrer antes ou durante o transporte. Essas injúrias contribuem para o estabelecimento dos patógenos que estão presentes nos frutos ou nas embalagens provenientes do campo de produção (Seberry \& Harris (17); Thompson (20); Medina et al. (13); Alves et al. (2)).

$\mathrm{O}$ estabelecimento dos patógenos pode ocorrer de diferentes formas, infecção quiescente (q), ferimentos (f) ou em pré-colheita (pc), e contribuir para a predominância de patógenos, em determinadas regiões. Essas formas de infecção são apresentadas por vários fungos patogênicos associados a frutos de banana, como Colletotrichum (q), Alternaria (q), Thielaviopsis (f), Phomopsis (q), Phytophthora (q/pc), Rhyzopus (f), Botrytis (q/pc), Deightoniella (pc), Fusarium (f/pc), Phyllosticta (pc), Mycosphaerella (pc), Nigrospora (f), Trichothecium (f), e Verticilliun (q) (Simmonds (18); Johnson \& Sanghote (8)).

No Brasil, a antracnose e a podridão da coroa são as principais doenças que ocorrem em pós-colheita de bananas e um dos principais fatores que afetam a qualidade dos frutos. Outros patógenos também estão associados a essas podridões em diferentes locais e épocas do ano, como Thielaviopsis paradoxa (Ceratocystes paradoxa), Botryodiplodia theobromae e Verticillium theobromae, causando podridão do engaço e podridão do fruto e a ponta de charuto, respectivamente (Alves et al. (2) e Medina et al. (13)).

Vários outros fungos foram detectados associados a podridões em banana, Penicillium, Alternaria triticina, Cladosporium oxysporium, Acremonium spp., Fusarium spp. e Eupenicillium (Jones (9)), Acremonium strictum, Alternaria sp., Cladosporium sp., Curvularia brachyspora, Pestalotiopsis cruenta, Phoma joliana, Verticillium theobromae, Aspergillus niger, A. flavus e A. fumigatus (Mesturino (14)).

Diante da diversidade de fungos, patogênicos ou não, associados a frutos de banana em pós-colheita, os objetivos deste trabalho foram avaliar a incidência dos fungos em banana 'Prata anã', provenientes do norte de Minas Gerais, e determinar a doença predominante na região.

\section{MATERIALEMÉTODOS}

O isolamento e a identificação dos fungos foram realizados no Laboratório de Fitopatologia da Universidade Federal de Viçosa, em Viçosa, Minas Gerais, no período compreendido entre os meses de fevereiro e março de 1998.

\section{Material Vegetal}

Os cachos de banana 'Prata anã' foram colhidos, em estádio pré-climatérico, de plantações comerciais de dois anos de idade no município de Jaíba, Minas Gerais. Os cachos foram despencados e as pencas selecionadas, acondicionadas em caixas plásticas e transportadas por caminhão, em doze horas, até a Universidade Federal de Viçosa. Na seleção, optou-se pelas pencas centrais dos cachos, rejeitando-se as duas pencas proximais e as três pencas distais, visando maior uniformidade dos frutos durante a maturação em pós-colheita.

\section{Métodos de Isolamento \\ Isolamento por Diluição em Placas a partir de Frutos} Verdes

Quarenta frutos colhidos em estádio pré-climatérico foram escolhidos casualmente e lavados individualmente em $100 \mathrm{~mL}$ de água destilada e esterilizada. A suspensão resultante foi diluída para $10^{-3}$ e 1,0 mL dessa diluição foi distribuído, separadamente, em três placas de Petri, contendo ágar-água e antibiótico. As placas foram assim incubadas à temperatura ambiente $\left(24 \pm 2{ }^{\circ} \mathrm{C}\right)$, por sete dias, e as colônias desenvolvidas foram identificadas e quantificadas.

Isolamento Direto de Frutos durante o Amadurecimento

Buquês colhidos em estádio pré-climatérico e contendo três frutos foram incubados sob condições ambientes de laboratório à temperatura de $24 \pm 2{ }^{\circ} \mathrm{C}$. Os fungos desenvolvidos na almofada, no meio e na extremidade distal dos frutos foram isolados diretamente dos frutos, durante os estádios de amadurecimento, identificados e quantificados. A incidência dos fungos foi avaliada até o completo amadurecimento dos frutos.

\section{Teste de Patogenicidade}

Buquês colhidos em estádio pré-climatérico e contendo três frutos foram lavados com água corrente, tratados com solução de hipoclorito de sódio a $1 \%$, durante três minutos, e lavados em água destilada esterilizada. Os fungos foram inoculados em frutos verdes pela substituição de discos da casca por discos de micélio (6,0 mm de diâmetro). Os discos de micélio foram provenientes de colônias monospóricas cultivadas em BDA + antibiótico, durante sete dias.

Os frutos foram inoculados individualmente na sua porção equatorial e lateral, em pontos de inoculação revestidos posteriormente com fita plástica transparente. Após a inoculação, os buquês foram acondicionados em câmara úmida, por vinte e quatro horas, e depois deixados amadurecer sob condições ambientes de laboratório. Outros buquês foram inoculados apenas com discos de BDA + antibiótico e serviram como tratamento testemunha.

As avaliações foram realizadas aos sete dias após a inoculação, quando os frutos estavam maduros, medindo-se a área média lesionada em torno do ponto de inoculação e calculada pelo produto do comprimento e largura da lesão. Os fungos inoculados foram reisolados e comparados com as colônias originais e as espécies confirmadas com auxílio da professora Maria Menezes, da Universidade Federal Rural de Pernambuco.

\section{RESULTADOS E DISCUSSÃO}

Colletotrichum musae, Trichoderma harzianum, Fusarium equisetii, Penicillium sp. Aspergillus parasiticus, Trichothecium roseum, Colletotrichum acutatum, Alternaria 
sp., Cladosporium musae e Curvularia lunata foram os fungos associados aos frutos (Figura 1). As espécies Cladosporium musae, Fusarium equisetii, Penicillium sp, Alternaria sp. e Colletotrichum acutatum foram as mais freqüentes, tanto em frutos verdes como em maduros, detectadas pelos dois métodos utilizados. Apenas Trichoderma harzianum, Curvularia lunata e Trichothecium roseum apresentaram freqüência abaixo de $5 \%$, tanto em frutos verdes, como em maduros (Figura 1).

Nos testes de patogenicidade, Colletotrichum musae apresentou maior área média lesionada com $5,8 \mathrm{~cm}^{2}$, enquanto para os demais fungos testados não passou de $1,50 \mathrm{~cm}^{2}$. Os fungos Colletotrichum musae, T. harzianum, F. equisetii, Penicillium sp., A. parasiticus, T. roseum, C. acutatum e Alternaria sp. foram patogênicos quando inoculados por ferimentos em frutos verdes. A patogenicidade dos fundos do gênero Colletotrichum, Fusarium, Trichoderma, Aspergillus e Penicillium foi confirmada por Ferrari (5), em frutos de banana.

Apesar de Colletotrichum musae ser considerado o agente primário da podridão de frutos de banana, outros fungos oportunistas aceleram a deterioração dos frutos a partir dessa infecção primária (Jones \& Slabaugh (10)). Os resultados mostraram que a presença dos fungos na superfície dos frutos, cuja patogenicidade foi confirmada ou não, podem promover o rápido desenvolvimento das podridões, que se estendem do fruto ao pedicelo e à coroa.

Nestes estudos, constatou-se a maior freqüência de Colletotrichum musae, seguido por Cladosporium musae, Penicillium sp., F. equisetii, Alternaria sp, C. acutatum e A.

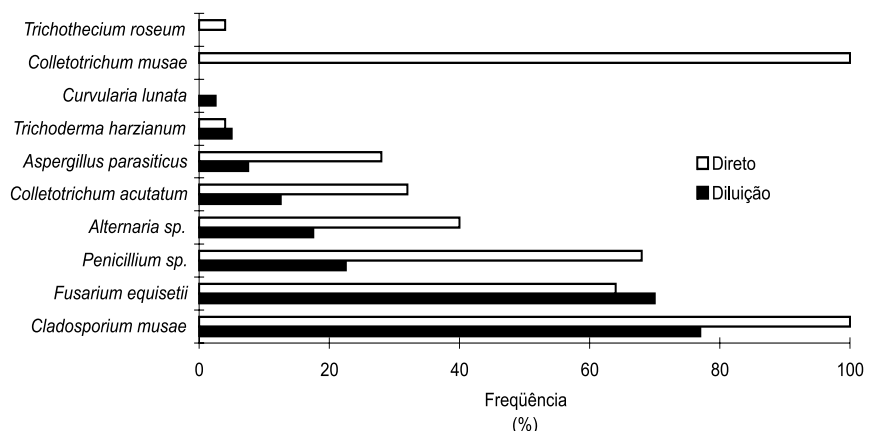

(\%)

B

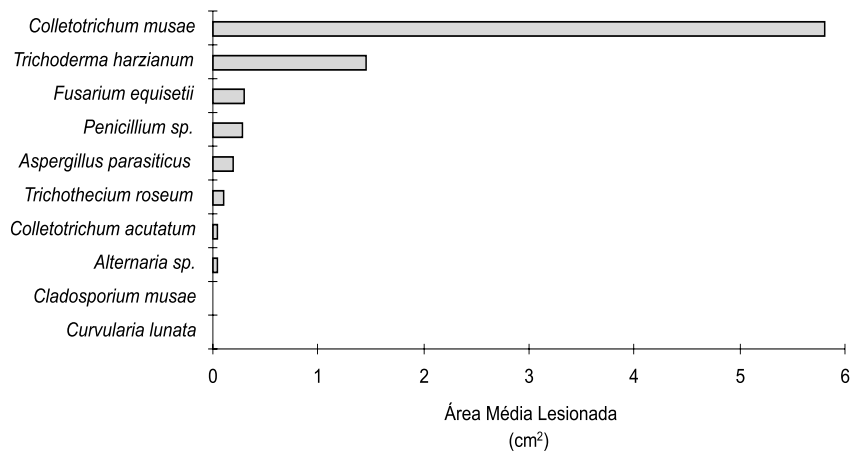

Figura 1. A - Frequiência de fungos isolados de frutos banana 'Prata anã' (AAB), utilizando dois métodos de isolamento; B - Área média lesionada, em torno do ponto de inoculação dos fungos isolados e inoculados em frutos de banana 'Prata anã', aos sete dias após a inoculação. parasiticus. O fungo Colletotrichum musae não foi detectado pelo método de diluição em placas, porém, por intermédio do método direto, foi encontrado em todos os frutos avaliados, exibindo sinais do patógeno e sintomas típicos da antracnose.

Esse fato deve-se, provavelmente, ao estádio inicial de infecção desse patógeno, que ocorre antes da colheita, durante o crescimento dos frutos, e se torna tipicamente quiescente ou latente até o inicio do amadurecimento (Simmonds (18) e Swinburn (19)). O processo infecioso envolve um período de vários meses, quando o patógeno se torna inativo ou quiescente, na forma de hifa subcuticular, logo após o início da infecção (Coates \& Gowanlock (4)). A reativação do crescimento do patógeno ocorre durante os processos de amadurecimento, quando mudanças fisiológicas ocorrem no tecido hospedeiro (Flaishman \& Kolattukudy (6); Prusky (16)).

Em estádio avançado de desenvolvimento da lesão, um estroma micelial compacto é formado entre a cutícula e o tecido necrosado, a partir do qual são produzidos os acérvulos contendo os conídios que são liberados pela ruptura da cutícula (Simmonds (18); Chau \& Alvarez (3); Swinburn (19)). O modo de infecção latente, causada por $C$. musae, parece favorecer, primeiramente, a colonização interna dos tecidos e, posteriormente, a ação dos fungos oportunistas que determinam as podridões nos frutos e na coroa.

Levantamentos da incidência de fungos em banana mostraram $C$. musae como o mais frequiente, seguido por Fusarium spp., F. pallidoroseum, B. theobromae e C. gloesporioides (Lutchmeah \& Santchurn (12)). O início do aparecimento dos sintomas das doenças mostrou-se mais relacionado com a duração do período de armazenamento do que com os estádios de maturidade dos frutos. As evidências foram mostradas quando frutos tratados com etileno não apresentaram nenhum sintoma aparente de doenças, devido ao rápido amadurecimento.

Dentre as espécies de Fusarium, a única detectada pelos dois métodos utilizados foi F. equisetii (Figura 1). Em outros estudos, Jimenez et al. (7) avaliaram a incidência e a patogenicidade de espécies de Fusarium em frutos de banana provenientes do Panamá, Equador e Ilhas Canárias. Constataram Fusarium semitectum (F. pallidoroseum) como a espécie predominante, seguida por $F$. moniliforme, F. solani, F. oxysporium e F. proliferatum. Fusarium subglutinans (G. fugikuroi), F. acuminatum (G. acuminata) e $F$. graminearum (G. zeae) mostraram-se mais agressivas, independentemente do local de sua ocorrência.

Neste estudo, a espécie $F$. equisetii mostrou-se agressiva, afetando, inclusive, frutos ainda verdes. As lesões provocadas por este patógeno mostraram-se limitadas ao redor do ponto de inoculação, indicando, provavelmente, a necessidade da associação com patógenos mais agressivos, como Colletotrichum musae.

Cladosporium musae foi o mais freqüentemente associado à superfície dos frutos, sendo detectado pelos dois métodos de isolamento utilizados, porém não se mostrou patogênico em frutos de banana. Este fungo é considerado um parasita fraco que causa doenças em folhas velhas de bananeiras, cultivadas em locais de elevada umidade. Essa doença se mostra de menor importância, apesar de as folhas afetadas secarem e caírem precocemente, comprometendo a produção (Ploetz et al. (15)). Entretanto, considerando o relato de se terem promovido sérias desfolhas na Tailândia e no Panamá, esse patógeno apresenta potencial para afetar as plantações no norte do Estado de Minas Gerais.

$\mathrm{O}$ estudo da ocorrência e freqüência de fungos em bananas 
pode fornecer informações adicionais sobre a presença de alguns gêneros que causam podridões em frutos e podem comprometer a saúde humana pela produção de micotoxinas, como Penicillium, Alternaria, Aspergillus, Fusarium e Phomopsis. Algumas dessas micotoxinas foram encontradas em produtos processados, como sucos de frutas.

\section{REFERÊNCIAS BIBLIOGRÁFICAS}

1. Agrios, G.N. Plant Pathology. San Diego: Academic Press, 1988. $803 p$.

2. Alves, E. J., Dantas, J. L. L., Soares Filho, W. S. Banana para exportação: aspectos técnicos da produção. Brasília: Embrapa. 1995. (Série de Publicações Técnicas: FRUPEX, 18).

3. Chau, K.F.; Alvarez, A. M. A. histological study of antracnose on Carica papaya. Phytopathology, v.73, p.1113-1116, 1983.

4. Coates, L.; Gowanlock, D. Infection process of Colletotrichum species in subtropical and tropical fruits. In: Postharvest handling of tropical fruits, 1994, Australia. Proceedings. Sidney: 1994, pp.162-168.

5. Ferrari, W.A; Microflora fúngica de frutos de bananeira no Distrito Federal. 1982. Tese (Mestrado). Universidade de Brasília, Brasília.

6. Flaishman, M.A; Kolattukudy, P.E. Timing of fungal invasion using host's ripening hormone as signal. Proc. Natl. Academic Science, USA, v.91, p.6579-6593, 1994.

7. Jimenez, M.; Loerieco, A; Bottaglio, A; Ocorrence and pathogenicity of Fusarium species in banana fruits. Journal of Phytopathology, v.137, p. 214-220, 1993.

8. Johnson, G. I.; Sangchote, S. Control post-harvest disease of tropical fruits: challanges for the 21st century. In: Postharvest handling of tropical fruits, 1994, Australia. Proceedings. Sidney: 1994, pp.140-161.

9. Jones, D.R. Chemical control of crown rot in Queensland bananas. Australian Journal of Exp. Agric., v. 31, p. 693-698, 1991.
10. Jones, D.R.; Slaubaugh. Banana disease caused by fungi: antracnose and fungal scald. In: R. C. Ploetz.; Zentmyer, W. T.; Nishigima, K. G.; Rohrback, H. D. Compendium of tropical fruits disease. Minessota: APS Press, 1994, p. 4-5.

11. Kays, S.J.; Postharvest physiology of perishable plant products. New York: Van noshard Reinhold. 1991.

12. Lutchmeah, R.S.; Santchurn, D. Common postharvest deterioration of banana cv. 'Naine' in Mauritius. Rev. Agricole et Sucrière de l'ile Maurice, v.70, p.28-36, 1991.

13. Medina, J.C.; Bleinroth, E, W.; Martin, Z.J. de; Travaglini, D. A.; Okada, M.; Quast, D.G.; Hashizume, T.; Moretti, V. A.; Neto, L. de C.B.; Almeida, L. A. S. B. de.; Renesto, O. V. Banana: cultura, matéria-prima, processamento e aspectos econômicos. Campinas: ITAL, 1995.

14. Mesturino, L. Disease of banana fruits: evaluation of alternative fungicides. Revista di Agricoltura Subtropicale e Tropicale, v.82, p.517-524, 1988.

15. Ploetz, R.; Zentmyer, G.A; Nishijima, W.T.; Rohrbach, K.G.; Ohr, H.D. Compendium of tropical fruits disease, Minessota-USA: APS Press, 1994.

16. Prusky, D. Pathogen quiescence in postharvest disease. Annual Review of Phytopathology, v.34, p.413-434, 1996.

17. Seberry, J. A.; Harris, D. R. Effects of plantation and post-harvest manegement factors on shelf life of 'Williams'banana. In: Postharvest handling of tropical fruits, 1994, Australia. Proceedings. Sidney: 1994, p.407-409.

18. Simmonds, J. H.; Latent infection in tropical fruits discussed in relation to the part played by species of Gloeosporium and Colletotrichum. Proccedings of the Royal Society of Queensland, Queensland, p. 92-120,1941.

19. Swinburn, T. R. Quiescent infections in post-harvest disease. In: Dennis, C., ed., Postharvest Pathology of Fruits and Vegetables. London: Academic Press, 1983. p.1-21.

20. Thompson, A K. Preharvest fungicidal spray for postharvest disease control. In: Postharvest handling of tropical fruits, 1994, Australia. Proceedings. Sidney: 1994, p. 169-171. 